\title{
INDÚSTRIA 4.0: ESTUDO DA ADERÊNCIA DE SEUS PRESSUPOSTOS AO CURSO DE ENGENHARIA MECATRÔNICA DE UM INSTITUTO FEDERAL DO NORDESTE BRASILEIRO
}

\author{
HeCTOR LEONARdo MOTA MOREIRA ${ }^{1}$ \\ ${ }^{1}$ Instituto Federal de Educação, Ciência e Tecnologia do Ceará - IFCE \\ $<$ dohector@gmail.com> \\ DOI: <https://doi.org/10.21439/conexoes.v15i0.1589>
}

\begin{abstract}
Resumo. O presente artigo trata do tema indústria 4.0 e do curso de Engenharia Mecatrônica ofertado por um Instituto Federal do nordeste brasileiro, com o objetivo de qualificar a aderência entre os conteúdos vistos ao longo do curso com os principais pressupostos da indústria 4.0. Trata-se de uma pesquisa quali-quanti descritiva, que se enquadra como estudo de caso. Após estudo bibliográfico acerca dos principais conceitos envolvendo a indústria 4.0 e seus pressupostos, selecionou-se uma gama de termoschave que foram, por meio de exaustiva busca, encontrados nas ementas das disciplinas que, supostamente, seriam as de maior proximidade aos pressupostos estudados. Com isso, questionou-se os docentes responsáveis pelo ensino dessas disciplinas por meio de um questionário, para qualificar essa relação entre variáveis com uma visão de indivíduos especializados. A partir dos dados obtidos, concluiu-se que, Sistemas Físico-Cibernéticos, Robótica Avançada e Manufatura Aditiva Híbrida são os pressupostos da indústria 4.0 que mais se encaixam no curso, enquanto outros como Big Data e Inteligência Artificial se encaixam de forma mais escassa. Além disso, uma lista de disciplinas relacionadas foi feita com base na menção dos ministrantes e concluiu-se que a maioria delas eram optativas, o que mostra que, as tecnologias da indústria 4.0 ainda não são prioridades no curso, mas cabe aos estudantes acolhê-las pois elas serão promissoras em um futuro próximo.
\end{abstract}

Palavras-chaves: Indústria 4.0. Automação. Engenharia Mecatrônica. Instituto Federal. Novas tecnologias.

\section{INDUSTRY 4.0: STUDY OF CORRESPONDING OF ITS PRESSUPOSTS TO MECHATRONICS ENGINEERING COURSE OF A FEDERAL INSTITUTE OF BRAZILLIAN NORTHEAST}

\begin{abstract}
This article deals with the theme of industry 4.0 and the course of Mechatronics Engineering offered by a Federal Institute of the Brazillian Northeast, with the objective of qualifying the adherence among the contents seen throughout the course with the main assumptions of the industry 4.0. This is a descriptive qualitative-quantitative research, which fits as a case study. After a bibliographical study about the main concepts involving the industry 4.0 and its assumptions, a range of key terms was selected that were, through exhaustive search, found in the menus of the disciplines that supposedly would be those of greater proximity to the assumptions studied. So, the professors responsible for teaching these subjects were questioned through a questionnaire, to qualify the relationship between these variables with a view of specialized individuals. From the data obtained, it was concluded that, Cyber-Physical Systems, Advanced Robotics and Hybrid Additive Manufacturing are the most important industry assumptions in the course of industry 4.0, while others such as Big Data and Artificial Intelligence fit more sparingly. In addition, a list of related subjects was made based on the lecturer's mention and it was concluded that most of them were optional, which shows that the technologies of industry 4.0 are not yet priorities in the course, but it is up to the students to welcome them, as they will be promising in the near future.
\end{abstract}

Keywords: Industry 4.0. Automation. Mechatronics Engineering. Federal Institute. New technologies.

\section{INTRODUÇÃO}

Nos últimos séculos, vivem-se, de tempos em tempos, as chamadas Revoluções Industriais. As três passadas revolucionaram a indústria de diversas formas, tornando a obsoleta produção manual em uma sofisticada produção autônoma, com máquinas fazendo o papel repetitivo que antes era feito pelos operários.

Segundo Dombrowski e Wagner (2014 apud OLI-
VEIRA; SIMÕES, 2017) um conjunto de inovações tecnológicas que ocasiona desdobramentos no âmbito social, político e econômico é denominado de revolução industrial. A Primeira Revolução Industrial ocorreu em meados do século XVIII, na Inglaterra, e introduziu as máquinas a vapor que seriam usadas nas fábricas produtoras de fios e tecidos, a indústria têxtil. Durante os séculos XIX e XX, o mundo viu uma ascensão de apa- 

INSTITUTO FEDERAL DO NORDESTE BRASILEIRO

relhos que se utilizavam da recém-descoberta energia elétrica, como rádios e telefones. Era a Segunda Revolução Industrial, que se utilizou também do petróleo para abastecer automóveis e aviões, que passaram a ser confeccionados. Por fim, a Terceira Revolução Industrial foi marcada pela computação e rápido compartilhamento de dados. A informação era a chave de sucesso de empresas que surgiam, como a Apple e a Microsoft.

Hodiernamente, está em acelerado ritmo de expansão a Quarta Revolução Industrial, que, também chamada de Indústria 4.0, tem como preceito a incorporação da digitalização à atividade industrial, acrescendo novos conceitos inovadores para a indústria, como a inteligência artificial, os sistemas ciber-físicos, o Big Data, a internet das coisas, a robótica, entre outros (CNI, 2016).

Nesse contexto, tem-se uma nova indústria em ascensão, modernizada e inovadora, e, com base nisso, o Brasil deve-se modernizar em paralelo com as grandes multinacionais. Para isso, é preciso formar profissionais capacitados, e que tenham familiaridade com a quarta revolução industrial.

Dessa forma, esse artigo tem como objetivo analisar o curso de Engenharia Mecatrônica, ofertado por um Instituto Federal do Nordeste Brasileiro, com a finalidade de verificar se as disciplinas curriculares estão no contexto da indústria 4.0, e com quais conceitos elas se associam.

Além desta seção introdutória, este artigo está estruturado da seguinte forma: a seção 2 trata da indústria 4.0 e seus principais pressupostos abordados; a seção 3 apresenta o conceito de mecatrônica como área e como curso de engenharia, além do caso estudado (curso de um Instituto Federal); a seção 4 trata da metodologia utilizada e as seções 5, 6 e 7 tratam dos resultados obtidos, conclusão e das referências bibliográficas, respectivamente.

\section{A INDÚSTRIA 4.0}

O conceito de "Indústria 4.0" surgiu na Feira de Hannover (Alemanha) de 2011, para descrever uma revolução que vem alterando organizações mundiais com o advento da digitalização industrial. Consistindo na cooperação entre sistemas físicos e virtuais de fabricação, a indústria 4.0 permite fábricas inteligentes e flexíveis com a criação de novos modelos operacionais, sustentados por uma gama de novas tecnologias (SCHWAB 2016).

No entanto, a quarta revolução industrial não diz respeito apenas à indústria inteligente, abrangendo áreas da biologia, computação quântica, nanotecnologia, energias renováveis e novos materiais, entre outros.
Para a realização deste artigo, serão abordados apenas os conceitos mais próximos da indústria e da engenharia mecatrônica.

Segundo (SCHWAB, 2016), há divergências se esse conceito seria, de fato, uma nova revolução industrial, ou apenas uma continuação das inovações decorrentes da terceira. Nesse contexto, três razões principais evidenciam que a Indústria 4.0 é única perante as três anteriores:

A. Acelerado ritmo de crescimento, de tal forma que cada vez mais novas tecnologias originam outras, em um processo que se repete constantemente, e em intervalos de tempo cada vez menores.

B. Novos conceitos estão surgindo e ganhando espaço no campo social e industrial, que aparecem como resultado dessa revolução.

C. Mudanças no sistema estão acontecendo em países, indústrias e sociedade.

Dessa forma, a sociedade atual tem por dever adequar-se o mais rápido possível a essas transformações. No campo do ensino, as instituições devem modificar seus conteúdos disciplinares, de modo a adequar os futuros profissionais da área da indústria e tecnologia às inovações desse campo, para que possam disputar o mercado de trabalho, cada vez mais qualificado. Outrossim, os trabalhadores devem adequar-se, tanto em formação acadêmica, como em competências como: criatividade, inovação, comunicação, solução de problemas e conhecimentos técnicos (AIRES; MOREIRA; FREIRE, 2017).

Quanto aos seus impactos, as tecnologias da indústria 4.0 mudam a relação entre serviços e sociedade, sendo possível pedir serviços de transporte e de entrega a partir de um simples smartphone. A relação entre clientes e fornecedor também foi alterada, uma vez que os clientes esperam um produto de maior qualidade e a empresa fornecedora tende a desenvolver produtos cada vez mais inteligentes. De certo, as indústrias procuram se tornar mais flexíveis e dinâmicas; os termos "smart factory", "intelligent factory" e "factory of future" surgem simbolizando essa vertente de fábricas que buscam se modernizar com equipamentos e cadeias de produção mais sofisticadas e inteligentes (COELHO, 2016).

No que diz respeito às fábricas brasileiras, segundo pesquisa da CNI, em 2016, o conhecimento sobre as tecnologias necessárias e sua incorporação na produção para a formação básica da indústria 4.0 era pouco difundido no país: $42 \%$ das empresas desconheciam sua importância e $52 \%$ não utilizavam ferramentas como manufatura aditiva, incorporação de serviços digitais nos 

INSTITUTO FEDERAL DO NORDESTE BRASILEIRO

produtos, automação digital sem sensores e projetos de manufatura por CAD/CAM.

Com foco em melhorar o processo de produção e aumentar a produtividade, o Brasil se mantém em um caminho positivo, porém limitado, pois deixa de lado oportunidades na exploração de novos modelos de negócios e desenvolvimento da cadeia produtiva. Apesar do rumo natural de foco em aumento da eficiência e em seguida a busca pelo desenvolvimento de novos produtos e modelos de negócios, na posição competitiva em que o país se encontra, o ideal seria que o movimento de digitalização fosse realizado, simultaneamente, em todas as dimensões.

Para se manter competitivo a nível global, é essencial que o Brasil tenha uma estratégia de incorporação das novas tecnologias em sua indústria. Há exemplos de países em que a indústria 4.0 já é foco da sua política industrial, o que leva a necessidade de não apenas buscar a incorporação das novas tecnologias, mas fazê-lo de forma veloz, afim de evitar o gap para seus competidores.

\subsection{Pressupostos da Indústria 4.0}

Há divergências entre especialistas quanto ao conceito de Indústria 4.0 em relação à uma quarta revolução industrial, mas é explícito que novas tecnologias apareceram nestes últimos anos, que implementaram a indústria e a sociedade como um todo. Nessa seção serão abordados alguns pressupostos da Indústria 4.0, pilares fundamentais para a seu desenvolvimento e fixação no mundo. São eles: a Inteligência Artificial, a Robótica Avançada, os Sistemas Físico-Cibernéticos, a Internet das Coisas, o Big Data e a Manufatura Aditiva/Híbrida.

\subsubsection{Inteligência Artificial}

Em um conceito simples, para Luger e Stubblefield (1993 apud LUSTOSA, 2010), a inteligência artificial (IA) é um ramo da ciência da computação que se dedica à automação de comportamento inteligente. Mas, por se tratar de inteligência artificial, é difícil considerar apenas uma definição. Sendo assim, ao longo do tempo ela adquiriu quatro linhas de pensamento:

A. Sistemas que pensam como seres humanos;

B. Sistemas que atuam como seres humanos;

C. Sistemas que pensam racionalmente;

D. Sistemas que atuam racionalmente.

As linhas de pensamento A e C referem-se ao processo de pensamento e raciocínio, enquanto que as linhas B e D referem-se às ações, o comportamento.
Além disso, as linhas de pensamento A e B comparam as ações da máquina com as possíveis ações humanas, enquanto as linhas C e D comparam com as ações humanas ideais, as que seriam consideradas racionais (GOMES, 2010). Um dos ramos da IA que está presente nas resoluções de problemas dos usuários comuns da internet, isto é, sem ser empresas ou indústrias, são os agentes inteligentes. Um agente é um sistema de computador que está situado em algum ambiente e é capaz de executar ações independentes de forma flexível em seu meio, a fim de cumprir seus objetivos de projeto. As características: reativo, pró-ativo e social, são suficientes para classificar um agente como inteligente (WOOLDRIDGE, 1999). Na indústria 4.0, a inteligência artificial pode ser usada de acordo com suas técnicas: evolutiva, conexionista, cognitiva e biológica. As mais usadas na indústria são a evolutiva e conexionista. Como aplicação na indústria, braços robóticos podem ser usados em uma linha de produção, apoiando e realizando tarefas repetitivas com precisão, tais como montagem de equipamentos e embalagem de produtos, evitando o desgaste humano pelas ações repetitivas. A inspeção visual de produtos em uma linha de produção por equipamento com inteligência artificial pode identificar produtos fora do padrão com rapidez e flexibilidade, diminuindo o erro humano.

\subsubsection{Robótica Avançada}

Segundo Othman et al. (2016), robôs desempenham um importante papel na indústria manufatureira moderna. Uma face essencial da indústria 4.0 são métodos de produção autônomos alimentados por robôs que podem executar tarefas de maneira inteligente, com foco em segurança, flexibilidade, versatilidade e colaboração com humanos. Na indústria 4.0, robôs e humanos trabalharão lado a lado, em tarefas interligadas e usando interfaces homem-máquina com sensores inteligentes. Para (SCHWAB, 2016), os avanços dos sensores permitem aos robôs uma melhor compreensão e resposta ao ambiente. Além disso, os robôs podem acessar informações remotas da nuvem, rompendo com a necessidade de programação prévia. Isso permite que o uso de robôs seja ampliado para abranger várias funções, como: produção, logística e distribuição de documentos.

\subsubsection{Sistema Físico-cibernético}

Para Lee (2008) os sistemas físicos-cibernéticos (Cyber-Physical Systems - CPS) são integrações de processos computacionais e físicos em que computadores embarcados monitoram e controlam, geralmente 

INSTITUTO FEDERAL DO NORDESTE BRASILEIRO

com feedback contínuo, processos físicos. De acordo com Bauernhansl (2017 apud HERMANN; PENTEK; OTTO, 2016), o desenvolvimento dos CPS é caracterizado por três fases. Na primeira geração dos CPS estão inclusas as tecnologias de identificação, como etiquetas de RFID, que permitem identificação única. A segunda geração dos CPS está munida de sensores e atuadores com um escopo de funções limitado. Os sistemas ciberfísicos da terceira geração se diferem por serem: capazes de armazenar e analisar dados, equipados com múltiplos sensores e atuadores e compatíveis com o funcionamento em rede.

\subsubsection{Internet das Coisas (Internet of Things - IoT)}

Segundo Deidmar, Sobreira e Lima (2017) a Internet das Coisas (Internet Of Things - IoT) se refere a uma revolução tecnológica que em breve conectará equipamentos como eletrodomésticos, meios de transporte, roupas e maçanetas conectadas à internet e a outros dispositivos, como computadores e smartphones. No dia a dia, tem-se o uso da conexão via Bluetooth, ou NFC (Near Field Communication), para troca de dados entre smartphones e notebooks, ou mesmo como uma forma de controlar remotamente algum aparelho eletrônico, como é o caso de algumas caixas de som que podem ser pareadas com o smartphone.

No contexto da indústria 4.0, a internet das coisas (no ramo industrial adota-se Industrial Internet Of Things - IIoT) torna-se um importante pilar devido à possibilidade de conexão entre máquinas por meio de sensores e dispositivos eletrônicos, o que permite e facilita a troca de dados em tempo real, como por exemplo, o monitoramento remoto, que permite que as empresas rastreiem onde estão e como são movimentados seus objetos, do outro lado, têm-se o consumidor, que pode acompanhar o seu pedido em tempo real, ajudando-a a tornar-se uma indústria inteligente.

\subsubsection{Big Data}

Para Taurion (2013) o Big Data representa não só uma grande quantidade de dados, mas um montante de dados que seja volumétrico e variado, além de verossímil e valoroso para o negócio. Esse conceito tem surgido nos últimos anos devido ao crescente número de informações que as empresas (ou indústrias) têm de processar de forma rápida e consistente. Isso gera uma constante preocupação com a segurança desses dados, principalmente para as empresas de grande e médio porte, uma vez que o banco de dados de uma instituição é o que passa a dar sustentação aos seus processos produtivos.
Entretanto, o Big Data, nos termos da indústria 4.0, não trata apenas das variáveis, mas relaciona esses dados entre si, que por meio de um algoritmo (Inteligência Artificial) tende a encontrar um padrão para essas informações, de forma a mitigar possíveis erros futuros. Nesse ponto, as fábricas se beneficiam do Big Data para lidar com a criação de novas variáveis todos os dias e relacioná-las da melhor maneira, agilizando e otimizando seus processos.

\subsubsection{Manufatura Aditiva e Híbrida}

De acordo com Volpato e Costa (2013) a manufatura aditiva consiste na impressão 3D. Esse processo é feito acrescentando material em perfis complexos. Esse meio possui vantagens comparado apenas com o processo de usinagem como: economia de material para produção, fabricação de peças mais complexas e precisão no acabamento.

Com o desenvolvimento dessa nova tecnologia e aperfeiçoamento das técnicas de usinagem houve a necessidade de combinar manufatura aditiva e usinagem para melhoria e facilidade de produção de peças em geral, que abrangem diversos setores. Com isso, a manufatura aditiva híbrida se tornou um dos processos mais inteligentes e sofisticados de produção.

Em outras palavras, a manufatura aditiva híbrida é o processo que une impressão 3D e técnicas de usinagem para fabricação de diversos tipos de produtos. No contexto da indústria 4.0, esse método é usado em conjunto com os sistemas ciber-físicos, usando de softwares de CAD/CAM para ter maior eficiência no projeto e um produto final de qualidade, seja ele: peça, componente, protótipo, entre outros.

\section{A MECATRÔNICA E O CURSO DO INSTI- TUTO FEDERAL EM QUESTÃO}

Para (ROSARIO, 2005), o termo mecatrônica foi utilizado pela primeira vez no Japão, no fim dos anos 70, como resultado da junção bem-sucedida entre mecânica, eletrônica e processos digitais na feitura de produtos de consumo. A mecatrônica pode ser compreendida como a interseção da engenharia mecânica, a engenharia elétrica (eletrônica) e o controle por computador no projeto e manufatura de produtos e processos. A sinergia entre as especialidades que compõe a mecatrônica possibilita vantagens, como as listadas na Tabela 1 .

Segundo Cetinkunt (2008), o antigo modelo para uma equipe de projeto de produtos eletromecânicos, incluía:

1. Engenheiro (s) que projetava (m) os componentes mecânicos de um produto. 


\begin{tabular}{|l|l|}
\hline \multicolumn{1}{|c|}{ Desenvolvimento de projeto } & \multicolumn{1}{c|}{ Desenvolvimento de produto } \\
\hline Simplificação do sistema mecânico; & Programabilidade (flexibilidade de operação); \\
Redução do tempo e custo de desenvolvimento; & Auto monitoramento e prevenção ativa de acidentes; \\
Facilidade de introduzir modificações ou novas capacidades; & Autodiagnostico em caso de falhas; \\
Flexibilidade para receber futuras modificações ou novas funcionalidades. & $\begin{array}{l}\text { Redução do custo de manutenção e do custo de energia; } \\
\text { Elevado grau de precisão e confiabilidade. }\end{array}$ \\
\hline
\end{tabular}

2. Engenheiro (s) que projetava (m) os componentes elétricos, a lógica e os algoritmos.

3. Engenheiro (s) que projetava (m) o hardware do computador e a implementação do software para controlar o produto em tempo real.

Um engenheiro mecatrônico é capaz de realizar todas essas funções. Além disso, para um projeto ótimo do produto, as etapas do projeto não são feitas sequencialmente, mas sim, de forma simultânea. A Mecatrônica não é um novo ramo da engenharia, mas representa o estado de evolução atual dos ramos base para projetos de sistemas eletromecânicos. O produto final do trabalho de um engenheiro mecatrônico é um protótipo funcional de um produto ou sistema eletromecânico controlado por um computador embarcado.

\subsection{A Formação em Mecatrônica}

Em meados da década de 80, países como Austrália, Japão e Coreia do Sul implementaram cursos de graduação e pós-graduação voltados ao ensino de mecatrônica. Nos Estados Unidos, disciplinas que apresentam o conceito de mecatrônica foram inseridas na grade curricular de vários cursos de engenharia.

No Brasil, os primeiros cursos de graduação em mecatrônica surgiram no fim da década de 80 e foram denominados pelo Ministério da Educação de curso de Engenharia de Controle e Automação, sendo implementados em diversas universidades e faculdades de tecnologia.

O caráter multidisciplinar de sua formação permite ao profissional de controle e automação elaborar estudos e projetos, assim como participar da direção e fiscalização de atividades relacionadas ao controle de processos e automação de sistemas industriais. Por ter cunho generalista, a formação do engenheiro de controle e automação requer um esforço notável dos corpos docente e discente, mas findará suprindo uma lacuna existente no mercado, provendo uma realização profissional interessante (ROSARIO, 2005).

Dito isso, tomaremos como base para este artigo o curso superior de Engenharia de Mecatrônica de um Instituto Federal do Nordeste Brasileiro que, em cinco anos (10 semestres), prepara profissionais para o desempenho de atividades ligadas ao controle e automação de sistemas a variáveis contínuas ou discretas, principalmente no ambiente industrial.

\section{METODOLOGIA APLICADA}

Para a realização deste artigo, foram feitas pesquisas bibliográficas acerca dos principais conceitos da indústria 4.0. A partir das pesquisas foram selecionados termos-chave que melhor representam cada um deles (ver Tabela 2). Esses termos-chave foram retirados da revisão bibliográfica feita de cada um desses conceitos, utilizando como critério: conceitos de maior relevância e maior frequência com que apareciam ao longo do texto.

A partir desses termos foram feitas buscas nos programas de componente curricular do curso de engenharia mecatrônica de um Instituto Federal do Nordeste do Brasil (70 disciplinas ao todo, ao longo do curso), através do software Adobe Acrobat Reader. Dessa forma, as disciplinas que tiveram suas ementas apresentando pelo menos um dos termos-chave, em um contexto relacionado à indústria 4.0, eram tidas como possíveis casos de contato dos alunos com aquele conceito da Quarta Revolução Industrial. Para cada uma dessas disciplinas, entrou-se em contato com o docente ministrante, que respondeu um questionário qualitativo acerca da correspondência do curso com cada um dos pressupostos da indústria 4.0. Além disso, o questionário ainda deixava em aberto para o docente em questão expor sua opinião sobre qual disciplina aborda cada conceito e um espaço para comentários. Do total de 70 disciplinas, retirou-se dez por serem propedêuticas e quatorze por não terem nenhum dos termos-chave em suas ementas. Por conseguinte, sobraram 46 disciplinas, todas técnicas, que eram ministradas por um total de vinte professores.

Devido à limitação de tempo para o término do artigo, foram consultados apenas doze docentes $(60 \%$ do total), dentre os quais, a majoritária parte ministra mais de uma disciplina para os estudantes de engenharia mecatrônica do campus.

No questionário, dividiu-se os seis pressupostos abordados no presente artigo em dezoito tópicos principais (três tópicos por pressuposto), para que os pro- 
Tabela 2: Pressupostos da Indústria 4.0 e termos-chave associados

\begin{tabular}{|l|l|}
\hline \multicolumn{1}{|c|}{ Conceito } & \multicolumn{1}{c|}{ Termos-chave } \\
\hline Inteligência Artificial & Agente inteligente, Aprendizado de máquina, Automação, Robô Racional, Sistemas. \\
\hline Robótica & Automação, Autônomo, Inteligência Artificial, Interface, Robô, Manufatura Inteligente, Produção, Smart. \\
\hline Sistema Físico-Cibernético & Atuador, CPS, Sistema, Ciber-Físico, Computacional, Computador, Controle, Embarcado, Integrado, Processo, Sensor. \\
\hline Internet das Coisas & Computação em nuvem, Controle remoto em dispositivos, Dispositivos Periféricos, Indústria Inteligente, Sistemas em Rede. \\
\hline Big Data & Armazenamento, Armazenar, Dados, Data, Informações, Processamento, Processar. \\
\hline Manufatura Aditiva e Híbrida & Economia de Matéria Prima, Impressão 3D, Manufatura, Produção de Peças Complexas, Prototipagem, Usinagem. \\
\hline
\end{tabular}

fessores qualificassem a relação, ou aderência, de cada tópico com o curso. Poderia ser qualificado em uma escala de 0 a 5 , onde 0 significa não relacionado e 5 significa demasiadamente relacionado. Ainda existia a opção Desconheço Informação (D.I) que não contribuiria para a qualificação. Ao lado da escala, havia um espaço para que fosse escrita uma disciplina relacionada àquele tópico, se fosse de conhecimento do questionado. Os tópicos foram selecionados a partir dos principais termos-chave utilizados anteriormente.

Dessa forma, os resultados qualitativos foram quantificados em uma pontuação, por tópico, onde a Pontuação Máxima Ideal (se todos os docentes tivessem dado grau de aderência máxima) seria de 60, a Pontuação Máxima Real (retirando-se a opção D.I, se todos os restantes tivessem dado nota máxima), e a Pontuação Efetiva Atingida (o somatório de pontos para aquele tópico). Além disso, uma tabela foi feita com as disciplinas citadas pelos questionados relacionadas com seus tópicos. Os resultados obtidos são abordados na seção seguinte.

\section{RESULTADOS}

Usando o questionário, foram entrevistados oito doutores, três mestres e um docente com especialização, dentre os quais, estavam o coordenador do curso e especialistas em várias áreas. Para este trabalho foram tratados todos com o mesmo peso, ou seja, a pontuação dada por um doutor não prevaleceu sobre a de um mestre, por exemplo.

\subsection{Resultados por Subtópico}

Analisando os subtópicos, notou-se uma maior aderência em Sensores, Projeto assistido por computador e Automação, que é área de maior foco do curso. Também é visível a carência nas áreas de armazenamento e processamento de dados, assim como na parte de Computação em Nuvem. A Tabela 3 mostra o percentual de aderência de cada subtópico:

Uma gama de disciplinas foi citada para cada subitem dos pressupostos. A Tabela 4 contém as cadeiras e a quantidade de menções dos professores:
Após computar os dados, verificou-se a média de aderência obtida para cada pressuposto, como mostra a Figura 1.

Figura 1: Percentual da média de aderência dos pressupostos ao curso estudado

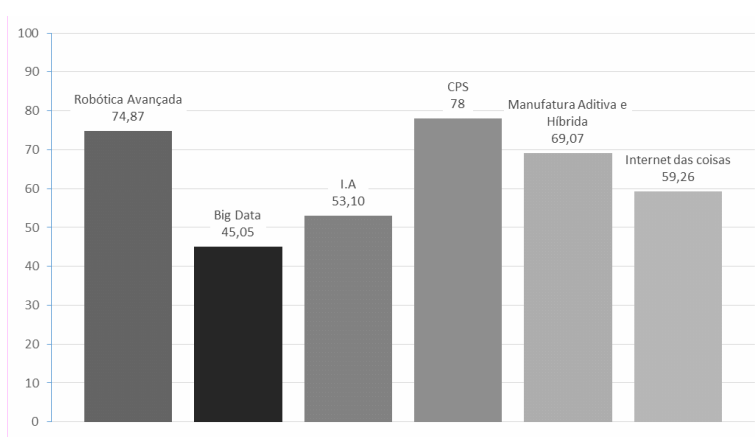

Por fim, teve-se que apenas $30 \%$ da totalidade de disciplinas ofertadas durante o curso de Engenharia Mecatrônica estudado foram relacionadas, pelos professores que responderam os questionários, aos pressupostos da Indústria 4.0. A Figura 2 mostra a distribuição total:

Figura 2: Distribuição das disciplinas

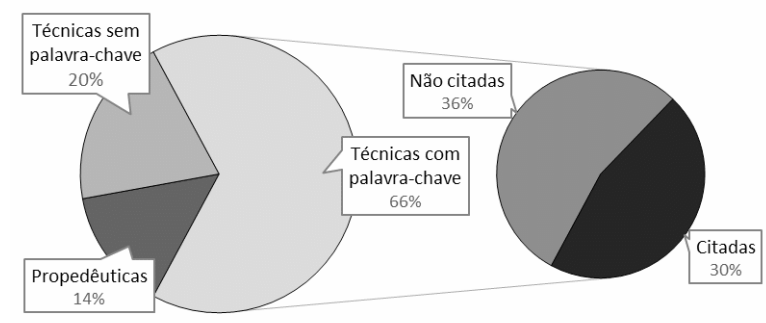

\section{CONCLUSÕES}

partir desses resultados, percebe-se que o curso de engenharia de mecatrônica em questão está, em parte, relacionado com os pressupostos da indústria 4.0. Se considerarmos $70 \%$ de aderência como "preparado", o 

INSTITUTO FEDERAL DO NORDESTE BRASILEIRO

Tabela 3: Percentual de aderência de cada subtópico.

\begin{tabular}{|c|c|c|c|}
\hline Pressupostos & $\begin{array}{l}\text { Máximo Possível } \\
\text { (excluindo D.I) }\end{array}$ & Somatório Real & $\begin{array}{l}\text { Nível de aderência } \\
\text { do subtópico }(\%)\end{array}$ \\
\hline \multicolumn{4}{|c|}{ Robótica Avançada } \\
\hline Robótica colaborativa & 35 & 23 & 65,71 \\
\hline Sensores & 45 & 40 & 88,89 \\
\hline Robôs autônomos & 40 & 28 & 70 \\
\hline \multicolumn{4}{|c|}{ Big Data } \\
\hline $\begin{array}{c}\text { Armazenamento } \\
\text { de dados }\end{array}$ & 45 & 18 & 40 \\
\hline $\begin{array}{c}\text { Processamento } \\
\text { de dados }\end{array}$ & 50 & 29 & 58 \\
\hline $\begin{array}{c}\text { Segurança } \\
\text { dos dados }\end{array}$ & 35 & 13 & 37,14 \\
\hline \multicolumn{4}{|c|}{ Inteligência Artificial } \\
\hline Robô racional & 30 & 12 & 40 \\
\hline Aprendizado de máquina & 40 & 26 & 65 \\
\hline Agente Inteligente & 35 & 19 & 54,29 \\
\hline \multicolumn{4}{|c|}{ Sistema Físico-cibernético } \\
\hline Automação & 50 & 43 & 86 \\
\hline Integração de sistemas & 30 & 24 & 80 \\
\hline Objeto inteligente & 25 & 17 & 68 \\
\hline \multicolumn{4}{|c|}{ Manufatura Aditiva } \\
\hline Impressão 3D & 40 & 22 & 55 \\
\hline $\begin{array}{l}\text { Projeto assistido } \\
\text { por computador }\end{array}$ & 55 & 46 & 83,64 \\
\hline Prototipagem & 35 & 24 & 68,57 \\
\hline \multicolumn{4}{|c|}{ Internet das Coisas } \\
\hline Computação em Nuvem & 40 & 16 & 40 \\
\hline Sistemas em rede & 45 & 31 & 68,89 \\
\hline $\begin{array}{c}\text { Controle de dispositivos } \\
\text { remotamente }\end{array}$ & 45 & 31 & 68,89 \\
\hline
\end{tabular}

curso se mostra preparado quando se trata de Sistemas Ciber-Fisicos, Robótica Avançada e Manufatura Aditiva Híbrida, o que era esperado devido ao conceito de mecatrônica e as áreas abrangentes. Entretanto, apesar do alto grau de aderência para a maioria dos pressupostos, as disciplinas citadas pelos docentes questionados eram, em grande parte, disciplinas optativas, o que mostra que as tecnologias e áreas abrangidas pela indústria 4.0 ainda não são prioridades no curso. Isso se deve principalmente pela baixa familiaridade das indústrias brasileiras (mercado de trabalho para os engenheiros em formação) às novas tecnologias.

O método de pesquisa utilizado se mostrou bastante efetivo. O primeiro método acusou as disciplinas técnicas que poderiam vir a ter relação com a indústria 4.0 de forma a eliminar, com razão, as disciplinas propedêuticas e as disciplinas técnicas que não teriam relação, o que facilitou a busca dos professores em um tempo adequado. Já o segundo método mostrou-se essencial para explicitar as disciplinas que de fato eram relacionadas (segundo a opinião de especialistas), além de qualificar a aderência do curso aos pressupostos de forma direta.

À luz dessas considerações, pôde-se ter uma visão mais ampla do curso e desenvolver um raciocínio crítico para as disciplinas opcionais do curso, que se mostraram importantes para lidar com as novas empresas que estão aderindo essas tecnologias. Para trabalhos futuros, pretende-se tratar os dados de forma mais criteriosa, ponderando as opiniões dos especialistas, além de falar com mais docentes especializados no curso e nos pressupostos abordados.

\section{REFERÊNCIAS}

AIRES, R. W. d. A.; MOREIRA, F. K.; FREIRE, 
INDÚSTRIA 4.0: ESTUDO DA ADERÊNCIA DE SEUS PRESSUPOSTOS AO CURSO DE ENGENHARIA MECATRÔNICA DE UM INSTITUTO FEDERAL DO NORDESTE BRASILEIRO

Tabela 4: Disciplinas mencionadas e quantidade de citações.

\begin{tabular}{|c|c|}
\hline Pressupostos & Disciplina relacionada (Número de citações) \\
\hline \multicolumn{2}{|r|}{ Robótica Avançada } \\
\hline Robótica colaborativa & Robótica I (5), Robótica II (6), Engenharia Assistida por Computador (1) \\
\hline Sensores & Instrumentação Eletrônica I (7), Instrumentação Eletrônica II (7) \\
\hline Robôs autônomos & Robótica I (4), Robótica II (7) \\
\hline \multicolumn{2}{|r|}{ Big Data } \\
\hline Armazenamento de dados & Microcontroladores (1), Dispositivos periféricos (1), Processamento Digital de Sinais (1) \\
\hline Processamento de dados & $\begin{array}{l}\text { Linguagem de Programação (2), Controle Digital (1), Automação Industrial (1), } \\
\text { Modelagem de Sistemas a Eventos Discretos (1) }\end{array}$ \\
\hline Segurança dos dados & NENHUMA \\
\hline \multicolumn{2}{|r|}{ Inteligência Artificial } \\
\hline Robô racional & Robótica I (2), Inteligência Computacional (1), Visão Computacional (1) \\
\hline Aprendizado de máquina & Robótica I (2), Inteligência Computacional (5) \\
\hline Agente Inteligente & Robótica II (2), Inteligência Computacional (4) \\
\hline \multicolumn{2}{|r|}{ Sistema Físico-cibernético } \\
\hline Automação & $\begin{array}{l}\text { Automação Industrial (6), Controle Digital (1), Instrumentação Eletrônica I (1), } \\
\text { Instrumentação Eletrônica II (1), Microcontroladores (2), Mecânica das Máquinas (1), } \\
\text { Sistemas Mecânicos (1), CAD (1), Dispositivos Periféricos (1) }\end{array}$ \\
\hline Integração de sistemas & Laboratório de Automação Industrial (1) \\
\hline Objeto inteligente & Visão Computacional (1) \\
\hline \multicolumn{2}{|r|}{ Manufatura Aditiva } \\
\hline Impressão 3D & $\begin{array}{l}\text { CAD (4), CAM/CNC/CIM (4), Engenharia Assistida por Computador (2), } \\
\text { Ciência dos Materiais (3), Desenho Técnico Mecânico (2) }\end{array}$ \\
\hline Projeto assistido por computador & CAD (6), CAM/CNC/CIM (2), Engenharia Assistida por Computador (2) \\
\hline Prototipagem & CAD (1), CAM/CNC/CIM (2), Engenharia Assistida por Computador (1) \\
\hline \multicolumn{2}{|r|}{ Internet das Coisas } \\
\hline Computação em Nuvem & Instrumentação Eletrônica I (1), Visão Computacional (1) \\
\hline Sistemas em rede & Automação Industrial (3), Instrumentação Eletrônica I (1) \\
\hline Controle de dispositivos remotamente & $\begin{array}{l}\text { Automação Industrial (1), Instrumentação Eletrônica I (1), Dispositivos Periféricos (1), } \\
\text { Processamento Digital de Sinais (1) }\end{array}$ \\
\hline
\end{tabular}

P. de S. Indústria 4.0: Competências requeridas aos profissionais da quarta revolução industrial. In: Anais do Congresso Internacional de Conhecimento e Inovação-ciki. Foz do Iguaçu: [s.n.], 2017. v. 1, n. 1, p. 1-15.

BAUERNHANSL, T. Die vierte industrielle revolution-der weg in ein wertschaffendes produktionsparadigma. In: BAUERNHANSL, T.; HOMPEL, M. T.; VOGEL-HEUSER, B. (Ed.).

Industrie 4.0 in Produktion, Automatisierung und Logistik: anwendung, technologie, migration. Wiesbaden: Springer, 2017. p. 1-31.

CETINKUNT, S. Mecatrônica. 1. ed. Rio de Janeiro: LTC, 2008.

CNI. Desafios para a indústria 4.0 no

Brasil. Brasília, 2016. Disponível em: $<$ https://bucket-gw-cni-static-cms-si.s3. amazonaws.com/media/filer_public/d6/cb/ d6cbfbba-4d7e-43a0-9784-86365061a366/desafios_ para_industria_40_no_brasil.pdf $>$. Acesso em: 18 mai. 2018.
COELHO, P. M. N. Rumo à Indústria 4.0. Dissertação (Mestrado em Engenharia e Gestão Ambiental) Faculdade de Ciências e Tecnologia, Universidade de Coimbra, Coimbra, 2016. 65 f.

DEIDMAR, G. L. C.; SOBREIRA, D. S.; LIMA, W. D. Internet das coisas na educação. Revista Tecnologias em Projeção, v. 8, n. 2, p. 78, 2017.

DOMBROWSKI, U.; WAGNER, T. Mental strain as field of action in the 4th industrial revolution. Procedia Cirp, Elsevier, v. 17, n. 47, p. 100-105, 2014.

GOMES, D. d. S. Inteligência artificial: conceitos e aplicações. Olhar Científico. v1, v. 1, n. 2, p. 234-246, 2010 .

HERMANN, M.; PENTEK, T.; OTTO, B. Design principles for industrie 4.0 scenarios. In: IEEE. 2016 49th Hawaii international conference on system sciences (HICSS). Koloa, 2016. p. 3928-3937.

LEE, E. A. Cyber physical systems: Design challenges. In: IEEE. 2008 11th IEEE international symposium on object and component-oriented real-time 
distributed computing (ISORC). Orlando, 2008. v. 1, p. 363-369.

LUGER, G. F.; STUBBLEFIELD, W. A. Artificial

Intelligence: Structures and Strategies for Complex

Problem Solving. 1. ed. Redwood City: Pearson, 1993.

LUSTOSA, V. G. O estado da arte em inteligência artificial. Colabor@-A Revista Digital da CVA-RICESU, v. 2, n. 8, p. 1, 2010.

OLIVEIRA, F. T.; SIMÕES, W. L. A indústria 4.0 e a produção no contexto dos estudantes da engenharia. In: UNIVERSIDADE FEDERAL DE GOIáS. Simpósio de Engenharia de Produção, Brasil. Catalão, 2017. p. 1-7.

OTHMAN, M. F.; AZLI, N. H. N.; TALIB, M. F.; BAHRIN, M. A. K. Industry 4.0: A review on industrial automation and robotic. Jurnal Teknologi, v. 78, n. 6-13, p. 137-143, 2016.

ROSARIO, J. M. Princípios de mecatrônica. 1. ed. São Paulo: Pearson Prentice Hall, 2005.

SCHWAB, K. A quarta revolução industrial. 1. ed. São Paulo: Edipro, 2016.

TAURION, C. Big Data. 1. ed. Rio de Janeiro: Brasport, 2013.

VOLPATO, N.; COSTA, C. A. Competências e recursos da rede de manufatura aditiva (rma) no brasil. In: Itatiaia: Associação Brasileira de Engenharia e Ciências Mecânica, $7^{\circ}$ Congresso Brasileiro de Engenharia de Fabricação. Itatiaia: [s.n.], 2013. p. 1.

WOOLDRIDGE, M. Intelligent agents. 1. ed. Cambridge: MIT Press Cambridge, 1999. 